\title{
FIRST RESULTS FROM GLAST-LAT INTEGRATED TOWERS COSMIC RAY DATA TAKING AND MONTECARLO COMPARISON
}

\author{
M. BRIGIDA, A.CALIANDRO, C.FAVUZZI, P.FUSCO, F.GARGANO, \\ F.GIORDANO, N.GIGLIETTO, F.LOPARCO, B.MARANGELLI, M.N.MAZZIOTTA, \\ N.MIRIZZI, S.RAINÒ, P.SPINELLI \\ for the GLAST LAT Collaboration
}

Dipartimento Interateneo di Fisica “M.Merlin” of Bari University \& INFN - BARI

Via Orabona 4,

70126 Bari, Italy

E-mail: monica.brigida@ba.infn.it

GLAST Large Area Telescope (LAT) is a gamma ray telescope instrumented with silicon-strip detector planes and sheets of converter, followed by a calorimeter (CAL) and surrounded by an anticoincidence system (ACD). This instrument is sensitive to gamma rays in the energy range between $20 \mathrm{MeV}$ and $300 \mathrm{GeV}$. At present, the first towers have been integrated and pre-launch data taking with cosmic ray muons is being performed.

The results from the data analysis carried out during LAT integration will be discussed and a comparison with the predictions from the MonteCarlo simulation will be shown.

\section{Introduction}

The Gamma-ray Large Area Space Telescope (GLAST) is an international and multi-agency mission planned for launch in 2007.

The instruments on the GLAST mission are the Large Area Telescope (LAT) and the GLAST Burst Monitor (GBM). The LAT will consist of three subsystems: a solid state detector (SSD) tracker (TKR), a CsI calorimeter, calorimeter (CAL) and a plastic scintillator anticoincidence (ACD) system. The combined detectors will observe the entire sky not occulted by Earth, with an energy coverage from a few $\mathrm{keV}$ to $300 \mathrm{GeV}$. The LAT has a modular structure, consisting of a $4 \times 4$ array of identical towers. Each tower is composed by a tracker, a calorimeter and data acquisition module. The tracking detector consists of layers of SSDs and tungsten converter foils[1,2].

At present, eight towers have been tested and assembled at Stanford Linear Accelerator Center (SLAC): the data collected during LAT integration give a good opportunity to test and validate the MonteCarlo (MC) simulation. 


\section{GLAST LAT MonteCarlo simulation}

The GLAST off-line software is manly based on GAUDI, a software architecture developed at CERN in order to support event data processing applications in different environments (from high level trigger in on-line system until off-line data analysis). GAUDI is a $\mathrm{C}++$ framework that implements the system architecture and ensures that the design features are respected, by following some general criteria in order to simplify data management. The main criteria adopted for the GAUDI design is a clear distinction between "data" objects and "algorithm" objects [3].

In the GLAST framework, GAUDI manages a series of algorithms applied to each generated particle to get the result of the complete simulation and reconstruction chain. The GLAST LAT simulation software implemented by the Collaboration is an Object-Oriented $\mathrm{C}++$ framework called Gleam (GLAST LAT Event Analysis Machine): the generation and interaction of generated particles with the detector is based on the Geant4 MonteCarlo toolkit. Fig. 1 shows the Gleam data flow. The output from the simulation is in the same format as the real data: the hits generated by the MC simulation are converted in digital output signal, as read by the electronics. Finally, the digitized events can be processed by the reconstruction package.

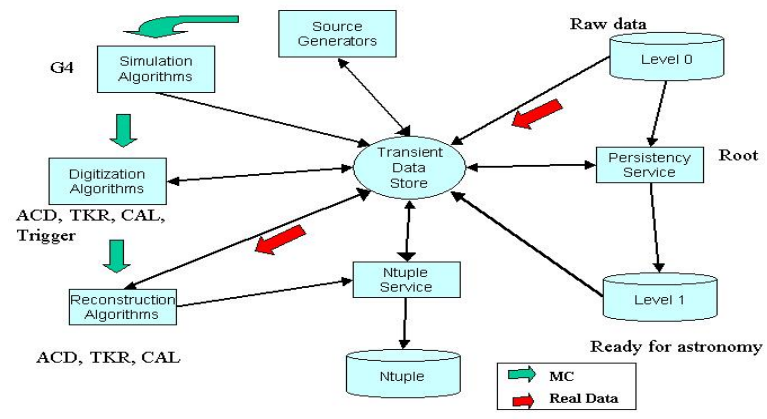

Figure 1. The Gleam Data flow.

To implement the Tracker digital output, a full simulation code has been developed [4]. The TKR Digit algorithm describe the physical processes that take place in a SSD, when crossed by an ionizing particle. The input parameters (hits) are provided by the Geant 4 LAT simulation. Then, the currents signals induced on each strip are evaluated and converted in voltage signals: the detector noise as well as the electronic noise are taken into account. Finally, the fired strips and the associated Time over Threshold (ToT) are evaluated [5].

In order to simulated the LAT behaviour during the integration stages, a dedicated version of Gleam tool (called EngineeringModel) has been adopted. 


\section{Overview of data taking during LAT integration}

Tests performed during hardware LAT integration are designed to ultimately calibrate the LAT, and to validate the MC simulations that will be used to verify the LAT science performance requirements. Data taking with cosmic rays and Van de Graaff photons has been performed [6]. At present data taking with 1, 2, 4, 6, 8 Modules (FMs) installed in the LAT grid are available.

Figure 1 shows the tower positions filled in some data taking configuration: the shaded squares indicate a tower installed in the grid. For each hardware configuration there is a baseline cosmic ray data-taking run for which the hardware is configured with nominal setting for ground analysis.

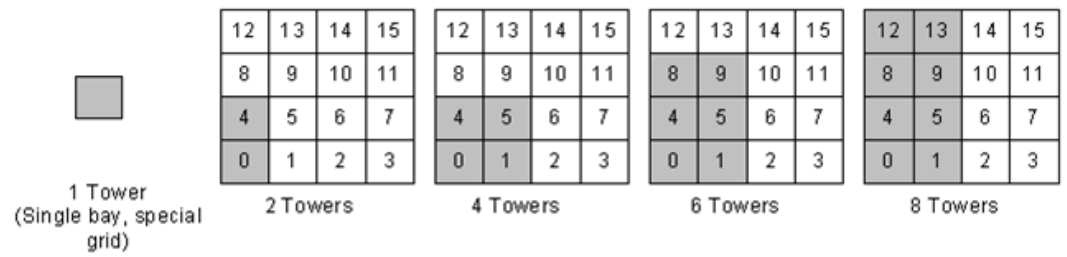

Figure 2. Towers in the grid during hardware integration.

\section{Data/MonteCarlo comparison}

In our analysis we used runs of cosmic ray data taking in eight towers configuration. We selected a data sample consisting of single muons tracks fully contained in a single TKR tower. The event selection has been performed by imposing following cuts:

- $\quad$ events triggered by the TKR;

- only a single muon tracks;

- events fully contained in a tower;

- minimum ionizing particles;

The data sample that we used in our analysis come from cosmic ray data taking at sea level, in standard hardware configuration, for a total of more than $2 \cdot 10^{6}$ events. Less than $10 \%$ of events survived to the cuts.

We studied the dependence of the hit strip multiplicity by the zenith angle $\theta$. As shown Fig.3, the hit multiplicity increases linearly with $1 / \cos \theta$, that is proportional to the track length in the SSDs: the plot shows a good agreement between data and MC predictions. 
In order to validate the MC digital output simulation we examined the ToTs distributions. Fig.4 shows the distribution of ToTs in each track layers for real muon data and the MC expectations for all integrated towers. As shown by the plot, the real data are well reproduced by the MC and the mean ToT value is consistent with expected charge deposition in $400 \mu \mathrm{m}$ thick silicon layers [4,5].

Finally, the dependence of the ToT in the track layers on the geometrical parameters $(\varphi$ and $\cos \theta)$ has been taken also in account.

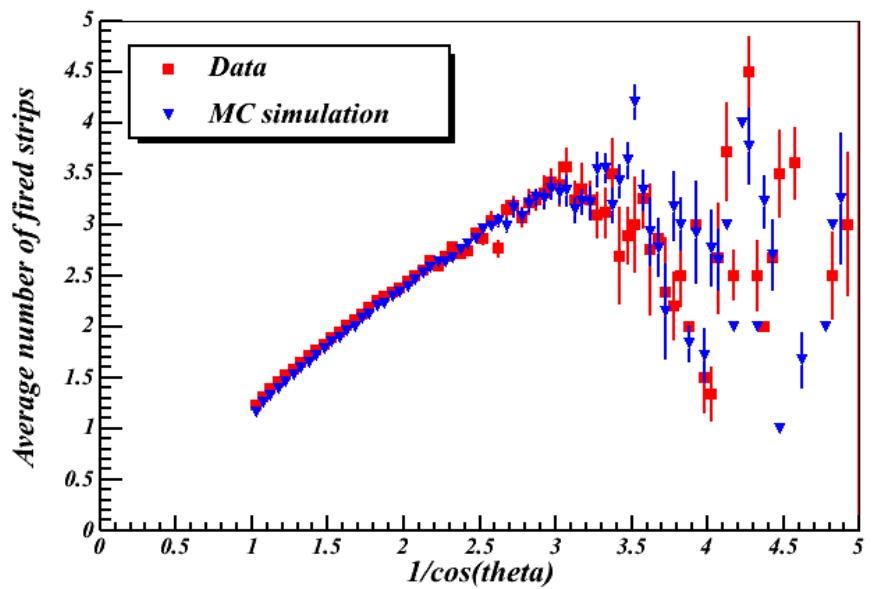

Figure 3. Average number of fired strips vs $1 / \cos \theta$ and MC comparison

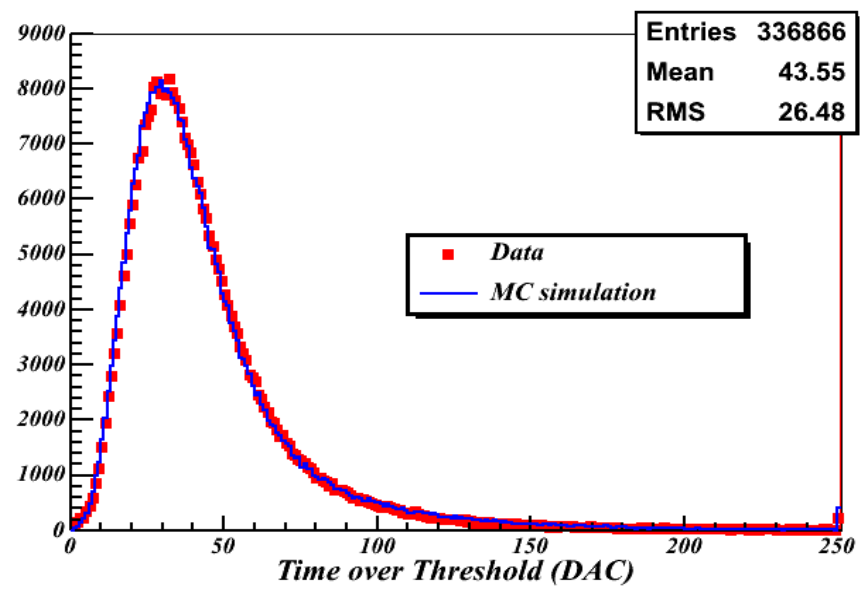

Figure 4. ToTs distribution for each track layers and MC comparison 


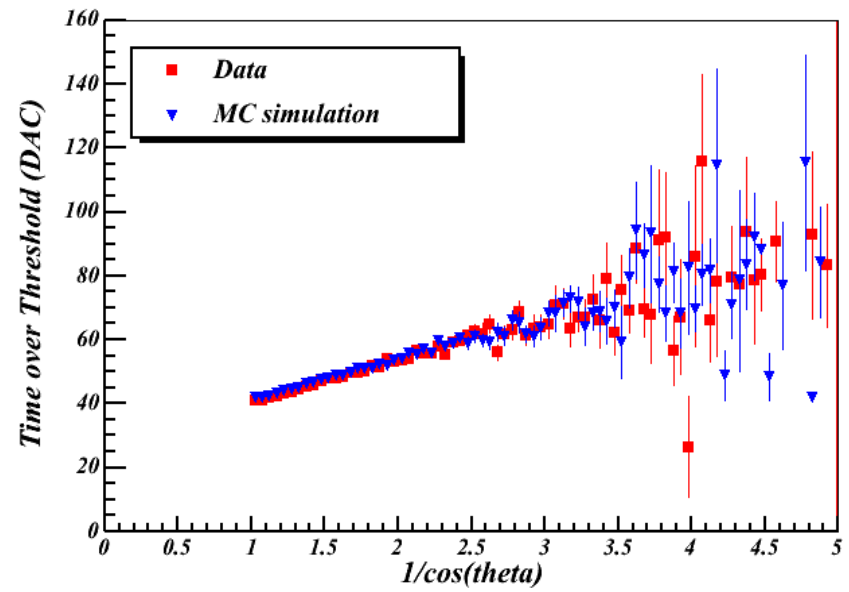

Figure 5. ToTs vs $1 / \cos \theta$ and MC comparison and MC comparison

As the hit strip multiplicity, the ToTs in the track layers increases linearly with the $1 / \cos \theta$ (proportional to the track length in the SSDs) as expected by the MC simulation (Fig.5).

\section{Conclusions}

The preliminary results from analysis of cosmic ray data taking during LAT integration are shown in this paper. The study of tracker behavior shows a good agreement between data and MC simulation predictions.

Cosmic rays test with the complete LAT configuration and beam test that will be performed during 2006 will be usefully to tune the Monte Carlo. After that, the GLAST Collaboration will be able to use the Monte Carlo simulations to predict the performance of the instrument.

\section{References}

1. http://glast.gsfc.nasa.gov/

2. http://www-glast.stanford.edu/pubfiles/proposals/bigprop/

3. http://proj-gaudi.web.cern.ch/proj-gaudi/welcome.html

4. M.N Mazziotta et al., Nuc. Instrum. And Meth. A533:322-343, 2004

5. M. Brigida et al. (2002), LAT internal note, LAT-TD-0105

6. Eduardo do Couto e Silva and Lee Steele (2005), LAT-TD-04631-02 